\title{
Correction to: $X$-coordinates of Pell equations as sums of two Tribonacci numbers
}

\author{
Eric F. Bravo ${ }^{1}$ - Carlos Alexis Gómez Ruiz ${ }^{1}$. Florian Luca ${ }^{2,3,4}$ \\ Published online: 19 December 2019 \\ ๑) Akadémiai Kiadó, Budapest, Hungary 2019
}

\section{Correction to: Periodica Mathematica Hungarica https://doi.org/10.1007/s10998-017-0226-8}

\author{
Abstract \\ In this work, we correct an oversight from [1].
}

\section{Introduction}

For a positive squarefree positive integer $d$ and the Pell equation $X^{2}-d Y^{2}= \pm 1$, where $X, Y \in \mathbb{Z}^{+}$, it is well known that all its solutions $(X, Y)$ have the form $X+Y \sqrt{d}=$ $X_{k}+Y_{k} \sqrt{d}=\left(X_{1}+Y_{1} \sqrt{d}\right)^{k}$ for some $k \in \mathbb{Z}^{+}$, where $\left(X_{1}, Y_{1}\right)$ is the smallest positive integer solution. Let $\left\{T_{n}\right\}_{n \geq 0}$ be the Tribonacci sequence given by $T_{0}=0, T_{1}=T_{2}=$ 1, $T_{n+3}=T_{n+2}+T_{n+1}+T_{n}$ for all $n \geq 0$. Let $U=\left\{T_{n}+T_{m}: n \geq m \geq 0\right\}$ be the set of non-negative integers which are sums of two Tribonacci numbers. In [1], we looked at Pell equations $X^{2}-d Y^{2}= \pm 1$ such that the containment $X_{\ell} \in U$ has at least two positive integer solutions $\ell$. The following result was proved.

Theorem 1.1 For each squarefree integer $d$, there is at most one positive integer $\ell$ such that $X_{\ell} \in U$ except for $d \in\{2,3,5,15,26\}$.

The original article can be found online at https://doi.org/10.1007/s10998-017-0226-8.

\footnotetext{
Carlos Alexis Gómez Ruiz

carlos.a.gomez@correounivalle.edu.co
}

Eric F. Bravo

eric.bravo@correounivalle.edu.co

Florian Luca

florian.luca@wits.ac.za

1 Departamento de Matemáticas, Universidad del Valle, Calle 13 No 100-00, Cali, Colombia

2 School of Mathematics, University of the Witwatersrand, Private Bag X3, Wits 2050, South Africa

3 Max Planck Institute for Mathematics, Vivatsgasse 7, 53111 Bonn, Germany

4 Department of Mathematics, Faculty of Sciences, University of Ostrava, 30 Dubna 22, 70103 Ostrava 1, Czech Republic 
Furthermore, for each $d \in\{2,3,5,15,26\}$, all solutions $\ell$ to $X_{\ell} \in U$ were given together with the representations of these $X_{\ell}$ 's as sums of two Tribonacci numbers. Unfortunately, there was an oversight in [1], which we now correct.

The following intermediate result is Lemma 4.1 in [1].

Lemma 1.2 Let $\left(m_{i}, n_{i}, \ell_{i}\right)$ be two solutions of $T_{m_{i}}+T_{n_{i}}=X_{\ell_{i}}$, with $0 \leq m_{i}<n_{i}$ for $i=1,2$ and $1 \leq \ell_{1}<\ell_{2}$. Then

$$
m_{1}<n_{1} \leq 1535, \quad \ell_{1} \leq 1070 \text { and } n_{2}<2.5 \cdot 10^{42} .
$$

The rest of the argument in [1] were just reductions of the above parameters. The first step of the reduction consisted in finding all the solutions to

$$
X_{\ell_{1}}=F_{n_{1}}+F_{m_{1}}, \quad \ell_{1} \in[1,1070] \quad 2 \leq m_{1}<n_{1} \leq 1535 .
$$

Unfortunately, the case $\ell_{1}=1$ was omitted in [1]. Here, we discuss the missed case $\ell_{1}=1$.

In order to reduce the above bound on $n_{2}$ from Lemma 1.2, we do not consider the equation $P_{\ell_{1}}^{ \pm}\left(X_{1}\right)=X_{1}$ since there is no polynomial equation to solve; instead, we consider each minimal solution $\delta:=\delta\left(X_{1}, \epsilon\right)$ of Pell equation $X^{2}-d Y^{2}=\epsilon= \pm 1$, for each $X_{1}=T_{m_{1}}+T_{n_{1}}$, according to the bounds in Lemma 1.2. Thus, after some reductions using the Baker-Davenport method on the linear form in logarithms $\Gamma_{1}$ and $\Gamma_{2}$ from [1, inequalities 3.9 and 3.12], for $(m, n, \ell)=\left(m_{2}, n_{2}, \ell_{2}\right)$, one shows that the only range for the variables to be considered is

$$
\ell_{1}=1, \quad 1 \leq m_{1}<n_{1} \leq 1811, \quad 1 \leq m_{2}<n_{2} \leq 3210, \quad \text { and } \quad 2 \leq \ell_{2} \leq 2220 .
$$

Now, with this new bound on $n_{2}$, by the same procedure (LLL algorithm and continued fractions) used on the linear form in logarithms $\Gamma_{3}, \Gamma_{4}$ and $\Gamma_{5}$ in [1, inequalities 3.15-3.26], we reduce again the bound on $n_{1}$ given in Lemma 1.2. Then, further cycles of reductions (for $n_{2}$ with the new bound of $n_{1}$ ) on $\Gamma_{1}$ and $\Gamma_{2}$ yield the following result.

Lemma 1.3 Let $\left(m_{i}, n_{i}, \ell_{i}\right)$ be two solutions of $T_{m_{i}}+T_{n_{i}}=X_{\ell_{i}}$, with $0 \leq m_{i}<n_{i}$ for $i=1$, 2. If $\ell_{1}=1$, then $1 \leq m_{1}<n_{1} \leq 160,1 \leq m_{2}<n_{2}<250$ and $2 \leq \ell_{2} \leq 175$.

An exhaustive search in this last range finds no new solutions. Hence, albeit the work in [1] missed one branch of computations which are described in this note, this does not affect the final result Theorem 1.1.

\section{Reference}

1. E.F. Bravo, C.A. Gómez, F. Luca, $X$-coordinates of Pell equations as sums of two Tribonacci numbers. Period. Math. Hung. 77(2), 175-190 (2018)

Publisher's Note Springer Nature remains neutral with regard to jurisdictional claims in published maps and institutional affiliations. 